\title{
Correlation between the area of high-signal intensity on SPIO-enhanced MR imaging and the pathologic size of sentinel node metastases in breast cancer patients with positive sentinel nodes
}

\author{
Kazuyoshi Motomura ${ }^{*}$, Tetsuta Izumi ${ }^{2}$, Souichirou Tateishi², Hiroshi Sumino², Atsushi Noguchi², \\ Takashi Horinouchi ${ }^{2}$ and Katsuyuki Nakanishi ${ }^{2}$
}

\begin{abstract}
Background: We previously demonstrated that superparamagnetic iron oxide (SPIO)-enhanced MR imaging is promising for the detection of metastases in sentinel nodes localized by CT-lymphography in patients with breast cancer. The purpose of this study was to determine the predictive criteria of the size of nodal metastases with SPIO-enhanced MR imaging in breast cancer, with histopathologic findings as reference standard.

Methods: This study included 150 patients with breast cancer. The patterns of SPIO uptake for positive sentinel nodes were classified into three; uniform high-signal intensity, partial high-signal intensity involving $\geq 50 \%$ of the node, and partial high-signal intensity involving $<50 \%$ of the node. Imaging results were correlated with histopathologic findings.

Results: Thirty-three pathologically positive sentinel nodes from 30 patients were evaluated. High-signal intensity patterns that were uniform or involved $\geq 50 \%$ of the node were observed in 23 nodes that contained macro-metastases and no node that contained micro-metastases, while high-signal intensity patterns involving $<50 \%$ of the node were observed in 2 nodes that contained macro-metastases and 8 nodes that contained micro-metastases. When the area of high-signal intensity was compared with the pathological size of the metastases, a pathologic $>2 \mathrm{~mm}$ sentinel node metastases correlated with the area of high-signal intensity, however, a pathologic $\leq 2 \mathrm{~mm}$ sentinel node metastases did not.

Conclusions: High-signal intensity patterns that are uniform or involve $\geq 50 \%$ of the node are features of nodes with macro-metastases. The area of high-signal intensity correlated with the pathological size of metastases for nodes with metastases $>2 \mathrm{~mm}$ in this series.
\end{abstract}

Keywords: Sentinel node, Breast cancer, Magnetic resonance imaging, Computed tomography, Superparamagnetic iron oxide, Nodal enhancement pattern, Lymph node metastasis

\section{Background}

Sentinel node biopsy is emerging as an alternative to axillary lymph node dissection for patients with breast cancer with clinically negative nodes [1-5]. It is associated with less morbidity, such as lymph edema and neuropathies, than axillary lymph node dissection, but with high

\footnotetext{
* Correspondence: motomurak@hotmail.com

'Department of Surgery, Osaka Medical Center for Cancer and Cardiovascular Diseases, 1-3-3 Nakamichi, Higashinari-ku, Osaka 537-8511, Japan Full list of author information is available at the end of the article
}

accuracy in the prediction of axillary nodal status; however, it still involves a surgical procedure with associated morbidity. Overall, $2-7 \%$ of patients are reported to have lymphedema even after sentinel node biopsy [6-8].

Recently, intravenously administered ultrasmall superparamagnetic iron oxide (USPIO)-enhanced MR imaging has been reported to be promising for the diagnosis of lymph node metastases as a noninvasive method. Some researchers have already evaluated nodal staging in various tumors [9-11]. Harisinghani et al. demonstrated a sensitivity 
of $100 \%$ with a specificity of $95.7 \%$ for nodal staging using USPIO-enhanced MR imaging in 80 patients with prostate cancer [9]. Rockall et al. reported that the sensitivity for nodal staging using USPIO-enhanced MR imaging in 768 lymph nodes from 44 patients with endometrial or cervical cancer was $100 \%$, significantly higher than the conventional method using size criteria, which has a sensitivity of $27 \%$ [10]. Stets et al. reported an accuracy of $87 \%$, sensitivity of $81 \%$, and specificity of $92 \%$ using USPIO-enhanced MR imaging for axillary lymph node metastases in 52 lymph nodes from 9 patients with breast cancer [11]. A recent meta-analysis demonstrated that USPIO-enhanced MR imaging is sensitive and specific, and superior to other modalities in the detection of nodal metastases for various malignancies [12].

We previously assessed MR imaging using interstitial injection of superparamagnetic iron oxide (SPIO) enhancement for the detection of metastases in sentinel nodes which were localized by CT-lymphography (CT-LG) in patients with breast cancer [13]. We demonstrated that SPIO-enhanced MR imaging accurately stages the axilla and may avoid even sentinel node biopsy in patients with breast cancer.

In the present study, we investigated the correlation between the area of high-signal intensity on SPIOenhanced MR imaging and the pathologic size of sentinel node metastases in breast cancer patients with pathologically positive sentinel nodes for determining the predictive criteria of the size of nodal metastases.

\section{Patients and methods}

\section{Patient selection}

One hundred and fifty consecutive patients with clinical T1-2 breast cancers and clinically negative nodes who underwent sentinel node biopsy at Osaka Medical Center for Cancer and Cardiovascular Diseases between January 2008 and November 2009 were enrolled in this study. Patients with nonpalpable breast cancer, prior axillary surgery or who were pregnant were excluded. Patients with a contraindication to CT or MR imaging, or a known allergy to the contrast agents were also excluded. The institutional review board of Osaka Medical Center for Cancer and Cardiovascular Diseases approved the study, and written consent was obtained from all patients.

\section{Sentinel node localization using CT-LG}

Interstitial CT-LG was performed using a multidetector row helical CT scanner (Light Speed VCT; GE Healthcare, Milwaukee, WI, USA). Contiguous 1.25-mm-thick CT images from the upper thorax to axillary regions were obtained once before administration of the contrast agent. CT scanning with a detector of $0.625 \mathrm{~mm}, 64$ rows was operated at $120 \mathrm{kV}, 300$ to 400 Auto-mA, $35 \mathrm{~cm}$ field of view, $512 \times 512$ matrix, section spacing of $1 \mathrm{~mm}$, and a table speed of $1.55 \mathrm{~mm} / 0.5 \mathrm{sec}$.

Transaxial CT images were reconstructed with a $1.25-\mathrm{mm}$ pitch and slice thickness of $0.3 \mathrm{~mm}$. Each patient was placed in the supine position. Three small plastic bullets were placed as landmarks on the upper chest wall on the skin for a merged image of the CT-LG and axial MR image. First, their arms were placed in an elevated position. After local anesthesia with a subcutaneous injection of $2 \mathrm{ml}$ of $2 \%$ procaine hydrochloride, $6 \mathrm{ml}$ iopamidol (Iopamiron 370; Bayer Schering Pharma, Osaka, Japan) was injected intradermally into the skin overlying the breast tumor and into the subareolar skin. A CT scan was performed after massaging the iopamidol injection site for one minute. Second, a CT scan was performed in the adducted arm position. Finally, their arms were placed in an elevated position again, and a localizing marker, which is usually used for CT-guided lung nodule biopsy, was attached to the skin at the axilla to identify the sentinel node location over the skin [14]. The sentinel node location was identified on the CT image and was indicated precisely by the crossing point of the localizing marker and the CT plane lights. The site was marked on the skin surface using an oil pen.

3D-CT images were reconstructed from the postcontrast CT images at each time point with volumerendering techniques and, if necessary, a workstation (GE Advantage Workstation, version 4.3; GE Healthcare, Milwaukee, WI, USA) was used to further examine lymph flow and the sentinel node (Figure 1a).

\section{MR imaging}

MR images were obtained using a $1.5 \mathrm{~T}$ imaging system (Sonata/Symphony; Siemens, Erlangen, Germany) with a 12-channel matrix body coil. T1-weighted axial images were obtained from the upper thorax to axillary lesions (repetition time in msec (TR), 140; echo time in msec (TE), 1.88; slice width, $4 \mathrm{~mm}$; interslice gap, $0 \mathrm{~mm}$; number of acquisitions, one; field of view, $28 \times 28 \mathrm{~cm}$; matrix, $141 \times 256)$; T2-weighted axial images were obtained through the axilla (TR, 4,000; TE, 85 effective time; echo train length, 11; slice width, $4 \mathrm{~mm}$; interslice gap, $0 \mathrm{~mm}$; number of acquisitions, one; field of view, $25 \times 25 \mathrm{~cm}$; matrix, $250 \times 384$ ). Additional nodal imaging sequences included T2*-weighted gradient echo images in the axial plane (TR, 613; TE, 30; flip angle, 30; slice width, $4 \mathrm{~mm}$; interslice gap, $0 \mathrm{~mm}$; number of acquisitions, one; field of view, $25 \times 25 \mathrm{~cm}$; matrix, $230 \times 384$ ).

Each patient was placed in the supine position in the adducted arm position. A $40 \mu \mathrm{l}$ aliquot of SPIO (Resovist; FUJIFILM RI Farma Co., Ltd., Kyobashi, Tokyo), containing $1.115 \mathrm{mg}$ iron, was diluted in $20 \mathrm{ml}$ normal saline. After local anesthesia with subcutaneous injection of $2 \mathrm{ml}$ of $2 \%$ procaine hydrochloride, $6 \mathrm{ml} \mathrm{SPIO}$, containing $0.3345 \mathrm{mg}$ iron, was injected intradermally into 


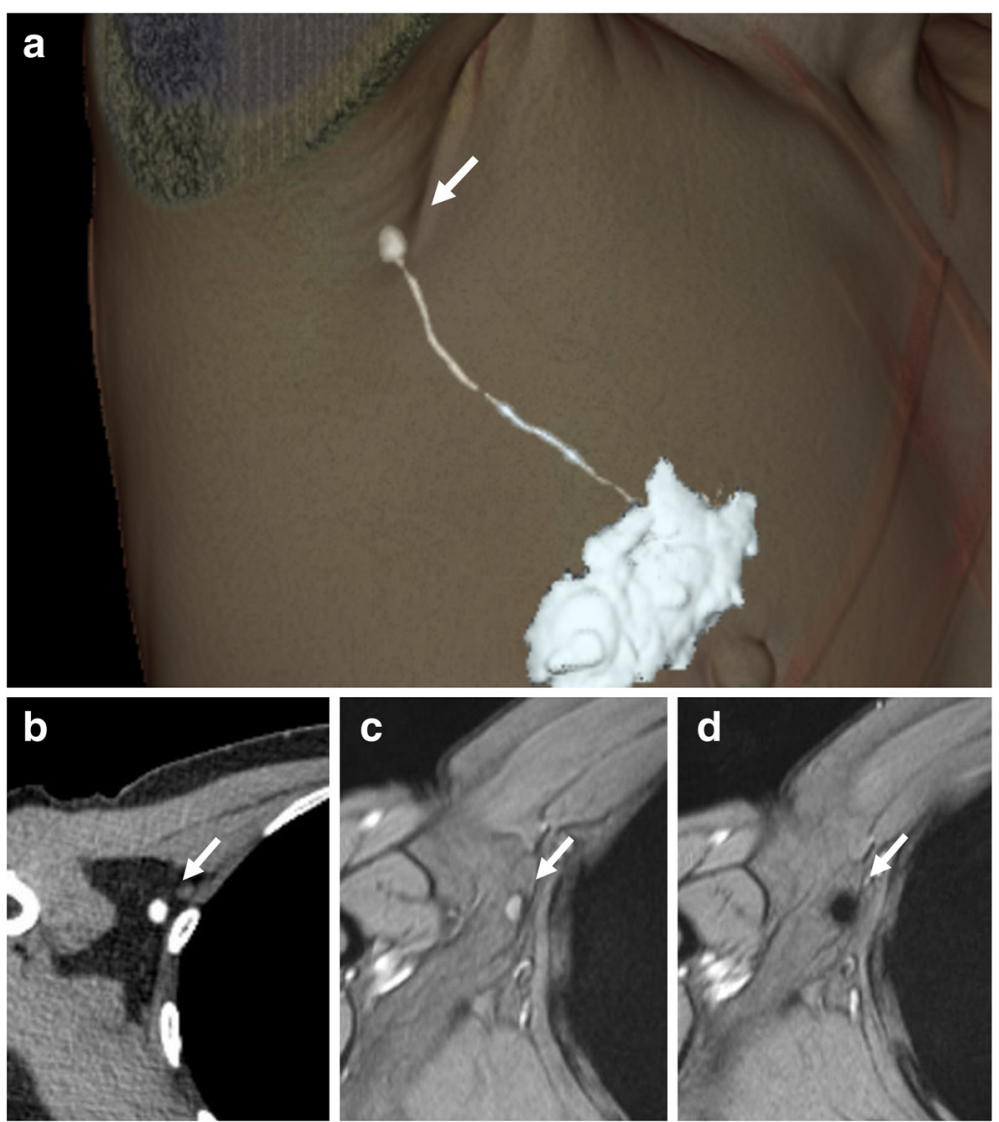

Figure 1 Sentinel node localization using CT-lymphography and SPIO-enhanced MR imaging for diagnosis. Three-dimensional CT-lymphography reconstructed from the first post-contrast images (a). Lymphatic vessels drained into a single axillary sentinel node (arrow). Images of CT-lymphography (b) and T2*-weighted axial MR images (c) at the same level were compared to specify the node (arrow) on T2*-weighted axial MR imaging corresponding to the sentinel node (arrow) identified by CT-lymphography. The node (arrow) showed high-signal intensity before administration of superparamagnetic iron oxide (SPIO). (d) After administration of SPIO, the node showed homogenous low signal intensity and was diagnosed as benign (arrow).

the skin overlying the breast tumor and into the subareolar skin. The injection sites of SPIO were gently massaged for one minute. At 18 to 24 hours after the administration of SPIO, the T1-, T2- and T2*-weighted sequences used for interpretation of the lymph node status were repeated. The MR imaging interval was determined by referring to the report of the study using USPIO [15]. T1-weighted and T2-weighted images were used for anatomic localization; the T1-weighted image in particular allows the shape and size of nodes clearly.

Images of the CT-LG (Figure 1b) and T2*-weighted axial MR images (Figure 1c) in the adducted arm position at the same level were compared to specify the node on T2*-weighted axial MR imaging corresponding to the sentinel node identified by CT-LG. If necessary, a merged image of the CT-LG and T2\%-weighted axial MR images was obtained on a workstation (PEGASYS; ADAC, Milpitas, CA, USA), with the help of small plastic bullets.
Nodes were evaluated on pre- and post-SPIO images by 1 reader (K.M.). Visual analysis was based on the diagnostic guidelines previously reported by Anzai et al. [16]. In brief, a node was considered non-metastatic if it showed homogenous low-signal intensity (Figure 1d) and metastatic if the entire node or more than $50 \%$ of the node has high-signal intensity on post-SPIO MR imaging compared with the signal intensity on pre-SPIO images. A node was considered possibly metastatic if less than $50 \%$ of the node has high-signal intensity [16]. In this study, patterns of SPIO uptake for positive sentinel nodes were classified into three referring to the guideline [16]; uniform high-signal intensity, partial high-signal intensity involving more than $50 \%$ of the node, and partial high-signal intensity involving less than $50 \%$ of the node (Figures 2, 3 and 4).

\section{Surgery}

Sentinel node biopsy was performed as described previously [17-19]. In brief, intradermal or intradermal and 

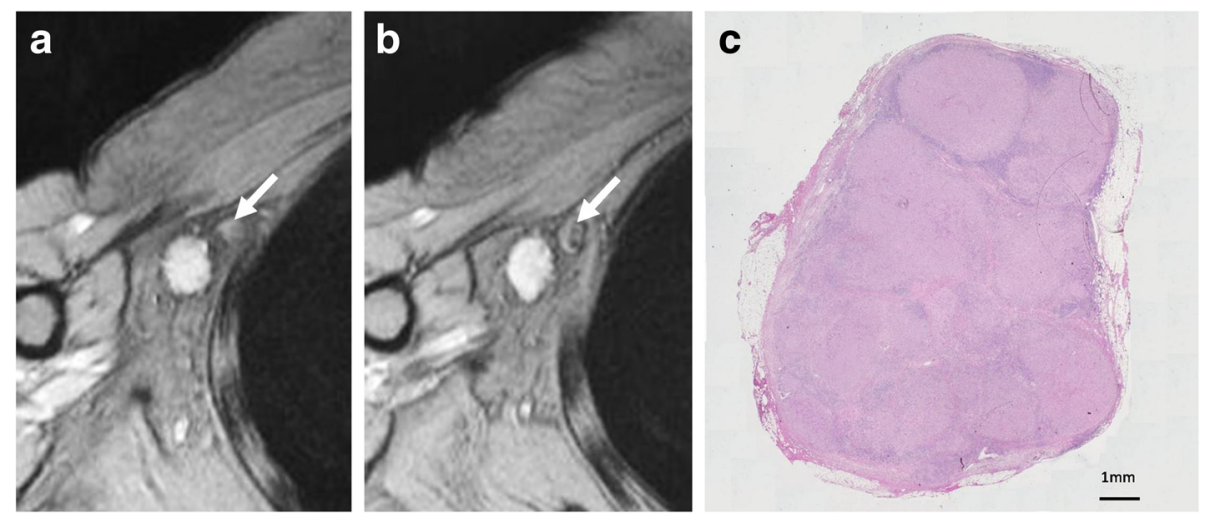

Figure 2 Pattern of uniform high-signal intensity on SPIO-enhanced MR imaging. (a) The node (arrow) showed high-signal intensity before administration of superparamagnetic iron oxide (SPIO). (b) After administration of SPIO, the node showed uniform high-signal intensity and was diagnosed as malignant (arrow). (c) Histological findings confirmed it as malignant. This node was almost entirely replaced by metastatic tissue.

subareolar injection of $0.3 \mathrm{ml}$ of $37 \mathrm{MBq}(1 \mathrm{mCi}) \mathrm{Tc}-99$ $\mathrm{m}$ tin colloid the day before surgery and peritumoral or intradermal and subareolar injection of $5 \mathrm{ml}$ indocyanine green (ICG, Diagnogreen 0.5\%; Daiichi Pharmaceutical Co. Ltd., Nihonbashi, Tokyo, Japan) 10 minutes before surgery were performed, and then the injection site was massaged manually for one minute. Lymphoscintigraphy was performed 2-3 hours after the radioisotope injection.

Breast surgery was performed before axillary surgery in all patients to minimize the influence of radioactivity from the injection site [17-19]. Hot nodes were identified using a gamma probe (neo2000; Neoprobe Corporation, Dublin, OH, USA). Dyed and/or hot nodes located just under the markers using CT images were defined as sentinel nodes and were removed.

\section{Histopathology}

Sentinel nodes were serially sectioned at $2 \mathrm{~mm}$ intervals. Hematoxylin and eosin sections of these nodes were prepared from each $2-\mathrm{mm}$ slice. When these nodes were tumor-negative in paraffin sections, an additional 4- $\mu \mathrm{m}$ section was cut and stained with immunohistochemistry (IHC) using the avidin-biotinylated peroxidase complex technique with the mouse monoclonal antibody against cytokeratin (NCL-CK19; Novocastra Laboratories Ltd., Newcastle, UK or AE1/AE3; Thermoelectron Corp., Waltham, MA, USA). On the basis of the 6th edition of the Union Internacional Contra la Cancrum TNM categories, metastatic nodes were categorized according to the degree of metastatic burden as follows: macrometastases $(>2 \mathrm{~mm})$ and micrometastases $(0.2$ to $2.0 \mathrm{~mm})$. Nodes with isolated tumor cells identified by IHC were considered to be
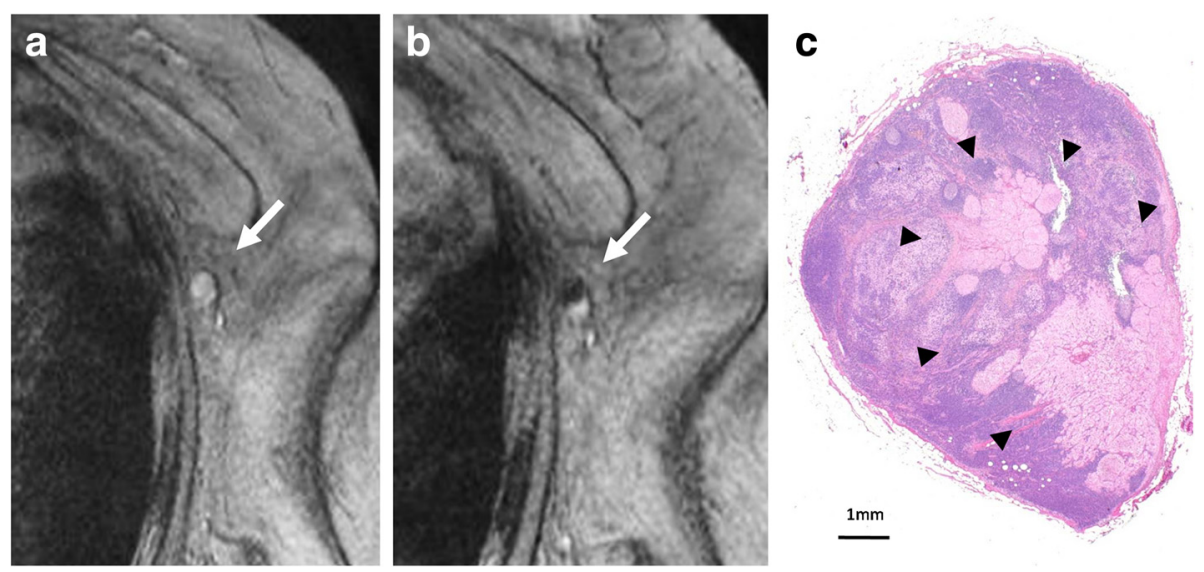

Figure 3 Pattern of partial high-signal intensity involving more than $\mathbf{5 0} \%$ of the node on SPIO-enhanced MR imaging. (a) The node showed high-signal intensity before administration of superparamagnetic iron oxide (SPIO). (b) After administration of SPIO, the node showed partial high-signal intensity involving more than $50 \%$ and was diagnosed as malignant (arrow). (c) Histological findings showed the presence of macro-metastases within the node (arrowheads). 
metastasis-negative [20]. The long axis of the area of high-signal intensity was compared with the pathological size of the metastases.

\section{Statistical analysis}

Spearman's rank-order correlation was used for statistical analysis.

\section{Results}

The mean age of the 150 patients was 56.0 (range, 3179) years and the mean pathologic tumor size was 19.4 (range, 0.2-60) $\mathrm{mm}$. Patient and tumor characteristics are summarized in Table 1 . The mean number of sentinel nodes identified by CT-LG was 1.15 (range 1-3). The mean size of sentinel nodes was 9.6 (range, 4-25) $\mathrm{mm}$. Thirty-three pathologically positive sentinel nodes from 30 patients were evaluated. Four false-negative patients were excluded. Three patterns of SPIO uptake were demonstrated for positive sentinel nodes. Six nodes (18.2\%) showed uniform high-signal intensity, 17 nodes (51.5\%) showed partial high-signal intensity involving more than $50 \%$ of the node, and 10 nodes (30.3\%) showed partial high-signal intensity involving less than $50 \%$ of the node. High-signal intensity patterns that were uniform or involved more than $50 \%$ of the node were observed in 23 nodes that contained macro-metastases and no node that contained micro-metastases, while high-signal intensity patterns involving less than $50 \%$ of the node were observed in 2 nodes that contained macro-metastases and 8 nodes that contained micro-metastases. When the area of high-signal intensity was compared with the pathological size of the metastases, a pathologic $>2 \mathrm{~mm}$ sentinel node metastases correlated with the area of high-signal intensity, however, a pathologic $\leq 2 \mathrm{~mm}$ sentinel node metastases did not. $\left(\mathrm{r}_{\mathrm{s}}=0.482, \mathrm{p}=0.015 ; \mathrm{r}_{\mathrm{s}}=0.309, \mathrm{p}=0.457\right.$, respectively $)$.

\section{Discussion}

The patterns of contrast enhancement have been demonstrated to distinguish between malignant and benign lymph nodes using USPIO-enhanced MR images [16]. A lymph node with an area of high-signal intensity encompassing the entire node or a portion of it was considered metastatic according to the diagnostic guidelines for USPIO-enhanced MR imaging, which are based on qualitative analysis of the results [16]. If there is no blackening of the node or if the node is hyperintense to surrounding tissue, or the node has central high-signal with darkening along the peripheral rim, or partial darkening whereby more than $50 \%$ of the node has an area of high-singal intensity, a node is diagnosed as metastatic. If less than $50 \%$ of the node has high-signal intensity, it is possibly metastatic. If the node has overall dark signal intensity, it is diagnosed as non-metastatic. A larger area of high-signal intensity within the node was reported to be more likely to be metastatic on USPIOenhanced MR imaging. Lahaye et al. reported that an estimated area of high-signal intensity within the node that was more than $30 \%$ was highly predictive of a metastatic node, with a sensitivity of $93 \%$ and a specificity of $96 \%$ in patients with primary rectal cancer [21]. They demonstrated that the most accurate and practical predictive criterion is the estimation of the percentage of high-signal intensity within the node on USPIOenhanced MR imaging. In the present study, we classified the patterns of SPIO uptake for positive sentinel nodes into three; uniform high-signal intensity, partial
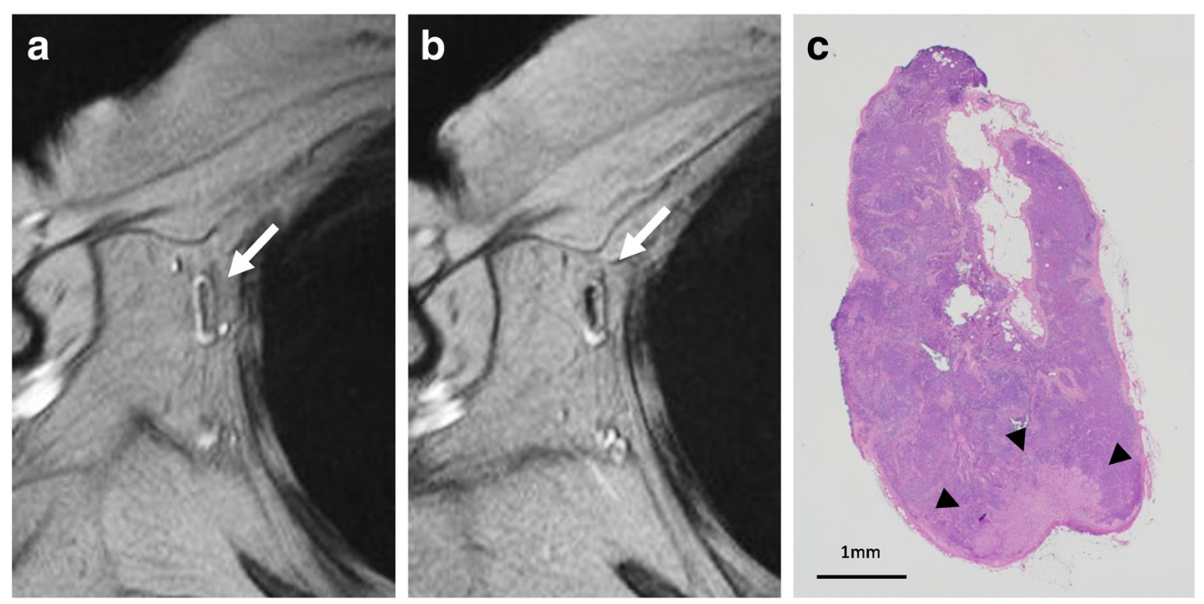

Figure 4 Pattern of partial high-signal intensity involving less than $50 \%$ of the node on SPIO-enhanced MR imaging. (a) The node showed high-signal intensity before administration of superparamagnetic iron oxide (SPIO). (b) After administration of SPIO, the node showed partial high-signal intensity involving less than 50\% and was diagnosed as malignant (arrow). (c) Histological findings showed the presence of micro-metastases within the node (arrowheads). 
Table 1 Patient characteristics

\begin{tabular}{|c|c|c|}
\hline & No. of patients & $\%$ \\
\hline \multicolumn{3}{|l|}{ Age, years } \\
\hline$<50$ & 45 & 30.0 \\
\hline$\geq 50$ & 105 & 70.0 \\
\hline \multicolumn{3}{|l|}{ Tumor size, cm } \\
\hline$\leq 2$ & 95 & 63.3 \\
\hline$>2, \leq 5$ & 53 & 35.3 \\
\hline$>5$ & 2 & 1.3 \\
\hline \multicolumn{3}{|l|}{ Tumor location } \\
\hline Upper outer & 82 & 54.7 \\
\hline Upper inner & 30 & 20.0 \\
\hline Lower outer & 24 & 16.0 \\
\hline Lower inner & 4 & 2.7 \\
\hline Central & 8 & 5.3 \\
\hline Multicentric & 2 & 1.3 \\
\hline \multicolumn{3}{|l|}{ Tumor histology } \\
\hline Invasive ductal & 132 & 88.0 \\
\hline Invasive lobular & 7 & 4.7 \\
\hline Ductal carcinoma in situ & 7 & 4.7 \\
\hline Others & 4 & 2.7 \\
\hline \multicolumn{3}{|l|}{ Type of surgery } \\
\hline Lumpectomy & 146 & 96.7 \\
\hline Mastectomy & 4 & 3.3 \\
\hline \multicolumn{3}{|l|}{ Estrogen receptor } \\
\hline Positive & 123 & 82.0 \\
\hline Negative & 27 & 18.0 \\
\hline \multicolumn{3}{|l|}{ HER-2/neu } \\
\hline Positive & 19 & 12.7 \\
\hline Negative & 131 & 87.3 \\
\hline
\end{tabular}

high-signal intensity involving more than $50 \%$ of the node, and partial high-signal intensity involving less than $50 \%$ of the node. We demonstrated that high-signal intensity patterns that were uniform or involved more than $50 \%$ of the node were observed in nodes with macro-metastases (Figures 2 and 3). High signal intensity patterns involving less than $50 \%$ of the node were often observed in nodes with micro-metastases (Figure 4). When the area of high-signal intensity was compared with the pathological size of the metastases, a pathologic $>2 \mathrm{~mm}$ sentinel node metastases correlated with the area of high-signal intensity, however, a pathologic $\leq 2 \mathrm{~mm}$ sentinel node metastases did not. The size of small metastatic foci could not be assessed because MR imaging had limited resolution in the present setting. It may be difficult to detect micro-metastases with a section thickness of $4 \mathrm{~mm}$ at this resolution on 1.5 $\mathrm{T}$ MR images. Interstitial administration of SPIO leads to excessive dosage or a high concentration of SPIO, which may conceal some micro-metastatic foci, resulting in underestimation of the size of metastatic foci, while the fatty hilum of a node, which coexists with metastases, may mimic a metastatic deposit, resulting in overestimation of the size of the metastatic foci. In our previous study, MR imaging with interstitial injection of SPIO was evaluated for the detection of metastases in sentinel nodes, which were localized by CT-LG in 102 patients with breast cancer [13]. The sensitivity, specificity, and accuracy of MR imaging for the diagnosis of sentinel node metastases were $84 \%, 91 \%$, and $89 \%$, respectively. In $40 \%$ of patients with micro-metastases, metastases were not detected, but all patients with macrometastases were successfully identified. False negatives may be due to micro-metastases. Of the 7 false-positive results, 6 were due to a prominent fatty hilum. False positives may be due to prominent fatty tissue and insufficient transition of SPIO to sentinel nodes. Fat-saturated images, a 3 T MR system and special coil for MR imaging may be needed to clearly identify small metastatic foci.

However, the clinical implication of micro-metastases is debatable. De Boer et al. reported that the presence of both isolated tumor cells and micro-metastases was associated with reduced disease-free survival among patients who did not receive systemic adjuvant therapy [22]. In patients with isolated tumor cells and micrometastases who received adjuvant therapy, disease-free survival was improved. In the systematic review, the presence of micro-metastases in axillary lymph nodes detected on single-section examination was associated with poorer disease-free and overall survival [23], while Hansen et al. reported that patients with micro-metastases do not have a worse disease-free or overall survival than sentinel nodenegative patients [24]. Whether intensive identification of the existence of small disease foci is needed in clinical practice is an urgent problem.

There were some limitations to our study. There was a relatively small number of metastatic nodes in our series. This was due to the selection of patients with T1-2 breast cancers and clinically negative nodes, who do not have many metastatic axillary nodes. In addition, it is unclear whether the $50 \%$ cut-off value was appropriate. The $50 \%$ cut-off value was applied according to the diagnostic guidelines for USPIO-enhanced MR imaging, and for high-signal intensity in which more than $50 \%$ of the node was observed in sentinel nodes with macro-metastases [16]. A larger study is needed to find the most appropriate cut-off value to confirm the results of our study.

\section{Conclusions}

High-signal intensity patterns that are uniform or involve more than $50 \%$ of the node are features of nodes with macro-metastases. The area of high-signal intensity 
correlated with the pathological size of metastases for nodes with metastases $>2 \mathrm{~mm}$ in this series.

\section{Abbreviations}

SPIO: Superparamagnetic iron oxide; USPIO: Ultrasmall superparamagnetic iron oxide; CT: Computed tomography; MR: Magnetic resonance; CTLG: Computed tomography-lymphography; TR: Repetition time; TE: Echo time; ICG: Indocyanine green; IHC: Immunohistochemistry.

\section{Competing interests}

The authors declare that they have no competing interests.

\section{Authors' contributions}

KM contributed to the conception and design of the study, data analysis and drafted the manuscript. TI and SH contributed the analysis and interpretation of the data of MR imaging. HS contributed the analysis and interpretation of the data of CT. AN provided methodological advice. TH and KN contributed to the conception and design of the study, analysis and interpretation of the data. All authors read and approved the final manuscript.

\section{Acknowledgments}

This work was supported in part by the National Cancer Center Research and Development Fund (22-38).

\section{Author details}

'Department of Surgery, Osaka Medical Center for Cancer and Cardiovascular Diseases, 1-3-3 Nakamichi, Higashinari-ku, Osaka 537-8511, Japan.

${ }^{2}$ Department of Radiology, Osaka Medical Center for Cancer and

Cardiovascular Diseases, Osaka, Japan.

Received: 1 March 2013 Accepted: 9 September 2013

Published: 13 September 2013

\section{References}

1. Giuliano AE, Kirgan DM, Guenther JM, Morton DL: Lymphatic mapping and sentinel lymphadenectomy for breast cancer. Ann Surg 1994, 220:391-401.

2. Giuliano $A E$, Jones RC, Brennan M, Statman R: Sentinel lymphadenectomy in breast cancer. J Clin Oncol 1997, 15:2345-2350.

3. Motomura K, Inaji H, Komoike Y, Kasugai T, Nagumo S, Noguchi S, Koyama $\mathrm{H}$ : Sentinel node biopsy in breast cancer patients with clinically negative lymph-nodes. Breast Cancer 1999, 6:259-262.

4. Krag D, Weaver D, Ashikaga T, Moffat F, Klimberg VS, Shriver C, Feldman S, Kusminsky R, Gadd M, Kuhn J, Harlow S, Beitsch P: The sentinel node in breast cancer: a multicenter validation study. N Engl J Med 1998 , 339:941-946.

5. Veronesi U, Paganelli G, Galimberti V, Viale G, Zurrida S, Bedoni M, Costa A, de Cicco C, Geraghty JG, Luini A, Sacchini V, Veronesi P: Sentinel-node biopsy to avoid axillary dissection in breast cancer with clinically negative lymph-nodes. Lancet 1997, 349:1864-1867.

6. Wilke LG, McCall LM, Posther KE, Whitworth PW, Reintgen DS, Leitch AM, Gabram SG, Lucci A, Cox CE, Hunt KK, Herndon JE 2nd, Giuliano AE: Surgical complications associated with sentinel lymph node biopsy: results from a prospective international cooperative group trial. Ann Surg Oncol 2006, 13:491-500.

7. Lucci A, McCall LM, Beitsch PD, Whitworth PW, Reintgen DS, Blumencranz PW, Leitch AM, Saha S, Hunt KK, Giuliano AE: American College of Surgeons Oncology Group: Surgical complications associated with sentinel lymph node dissection (SLND) plus axillary lymph node dissection compared with SLND alone in the American College of Surgeons Oncology Group Trial Z0011. J Clin Oncol 2007, 25:3657-3663.

8. McLaughlin SA, Wright MJ, Morris KT, Sampson MR, Brockway JP, Hurley KE, Riedel ER, Van Zee KJ: Prevalence of lymphedema in women with breast cancer 5 years after sentinel lymph node biopsy or axillary dissection: objective measurements. J Clin Oncol 2008, 26:5213-5219.

9. Harisinghani MG, Barentsz J, Hahn PF, Deserno WM, Tabatabaei S, van de Kaa $\mathrm{CH}$, de la Rosette J, Weissleder R: Noninvasive detection of clinically occult lymph-node metastases in prostate cancer. N Engl J Med 2003, 348:2491-2499.

10. Rockall AG, Sohaib SA, Harisinghani MG, Babar SA, Singh N, Jeyarajah AR, Oram DH, Jacobs IJ, Shepherd JH, Reznek RH: Diagnostic performance of nanoparticle-enhanced magnetic resonance imaging in the diagnosis of lymph node metastases in patients with endometrial and cervical cancer. J Clin Oncol 2005, 23:2813-2821.

11. Stets C, Brandt S, Wallis F, Buchmann J, Gilbert FJ, Heywang-Köbrunner SH: Axillary lymph node metastases: a statistical analysis of various parameters in MRI with USPIO. J Magn Reson Imaging 2002, 16:60-68.

12. Will O, Purkayastha S, Chan C, Athanasiou T, Darzi AW, Gedroyc W, Tekkis PP: Diagnostic precision of nanoparticle-enhanced MRI for lymph-node metastases: a meta-analysis. Lancet Oncol 2006, 7:52-60.

13. Motomura K, Ishitobi M, Komoike $Y$, Koyama H, Noguchi A, Sumino H, Kumatani $Y$, Inaji H, Horinouchi T, Nakanishi K: SPIO-enhanced magnetic resonance imaging for the detection of metastases in sentinel nodes localized by computed tomography lymphography in patients with breast cancer. Ann Surg Oncol 2011, 18:3422-3429.

14. Ohno Y, Hatabu H, Takenaka D, Higashino T, Watanabe H, Ohbayashi C, Sugimura K: CT-guided transthoracic needle aspiration biopsy of small ( $<$ or $=20 \mathrm{~mm}$ ) solitary pulmonary nodules. AJR Am J Roentgenol 2003, 180:1665-1669.

15. Hudgins PA, Anzai Y, Morris MR, Lucas MA: Ferumoxtran-10, a superparamagnetic iron oxide as a magnetic resonance enhancement agent for imaging lymph nodes: a phase 2 dose study. AJNR Am J Neuroradiol 2002, 23:649-656.

16. Anzai Y, Piccoli CW, Outwater EK, Stanford W, Bluemke DA, Nurenberg P, Saini S, Maravilla KR, Feldman DE, Schmiedl UP, Brunberg JA, Francis IR, Harms SE, Som PM, Tempany CM, Group: Evaluation of neck and body metastases to nodes with ferumoxtran 10-enhanced MR imaging: phase III safety and efficacy study. Radiology 2003, 228:777-788.

17. Motomura K, Inaji H, Komoike Y, Hasegawa Y, Kasugai T, Noguchi S, Koyama $\mathrm{H}$ : Combination technique is superior to dye alone in identification of the sentinel node in breast cancer patients. J Surg Oncol 2001, 76:95-99.

18. Motomura K, Komoike Y, Hasegawa Y, Kasugai T, Inaji H, Noguchi S, Koyama $\mathrm{H}$ : Intradermal radioisotope injection is superior to subdermal injection for the identification of the sentinel node in breast cancer patients. J Surg Oncol 2003, 82:91-96.

19. Motomura K, Nagumo S, Komoike $Y$, Koyama H, Inaji H: Accuracy of imprint cytology for intraoperative diagnosis of sentinel node metastases in breast cancer. Ann Surg 2008, 247:839-842.

20. American Joint Committee on Cancer, et al: Breast. In AJCC Cancer Staging Handbook. 6th edition. Edited by Greene FL, Page DL, Fleming ID, Fritz AG, Balch CM, Haller DG. New York: Springer; 2002:155-181.

21. Lahaye MJ, Engelen SM, Kessels AG, de Bruïne AP, von Meyenfeldt MF, van Engelshoven JM, van de Velde CJ, Beets GL, Beets-Tan RG: USPIO-enhanced MR imaging for nodal staging in patients with primary rectal cancer: predictive criteria. Radiology 2008, 246:804-811.

22. de Boer M, van Deurzen CH, van Dijck JA, Borm GF, van Diest PJ, Adang EM, Nortier JW, Rutgers EJ, Seynaeve C, Menke-Pluymers MB, Bult P, TjanHeijnen VC: Micrometastases or isolated tumor cells and the outcome of breast cancer. N Engl J Med 2009, 361:653-663.

23. de Boer M, van Dijck JA, Bult P, Borm GF, Tjan-Heijnen VC: Breast cancer prognosis lymph node metastases, isolated tumor cells, and micrometastases. J Natl Cancer Inst 2010, 102:410-425.

24. Hansen NM, Grube B, Ye X, Turner RR, Brenner RJ, Sim MS, Giuliano AE: Impact of in the sentinel node of patients with invasive breast cancer. J Clin Oncol 2009, 27:4679-4684.

doi:10.1186/1471-2342-13-32

Cite this article as: Motomura et al:: Correlation between the area of high-signal intensity on SPIO-enhanced MR imaging and the pathologic size of sentinel node metastases in breast cancer patients with positive sentinel nodes. BMC Medical Imaging 2013 13:32. 